\title{
Parents of newborns in the NICU enrolled in genome sequencing research: hopeful, but not naïve
}

\author{
Courtney Berrios, MSc, ScM ${ }^{1,2}$, Catherine Koertje, MD³, Janelle Noel-MacDonnell, PhD ${ }^{2,4}$, \\ Sarah Soden, $\mathrm{MD}^{1,2,3}$ and John Lantos, MD (i) ${ }^{2,5}$
}

\begin{abstract}
Purpose: In 2014, our institution launched a randomized controlled trial (RCT) comparing rapid genome sequencing (GS) to standard clinical evaluations of infants with suspected genetic disorders. This study aimed to understand parental response to the use of GS for their newborn babies.
\end{abstract}

Methods: Twenty-three of 128 parents whose infant had enrolled in the RCT completed a retrospective survey and interview addressing attitudes about GS and responses to receiving diagnostic information. We also collected information about participants' genetic literacy, genetic knowledge, numeracy, and symptoms of anxiety and depression.

Results: The majority reported positive $(13 ; 56.5 \%)$ or neutral 4 (4; $17.4 \%)$ feelings when approached about GS for their infant and $100 \%$ felt that GS was generally beneficial. The 12 participants who had received a unifying diagnosis for their child's symptoms described personal utility of the information. Some reported the diagnosis led to changes in medical care. Participants showed understanding of some of the psychological risks of GS. For example, 21 (91.3\%) agreed or strongly agreed that genetic testing could reveal disturbing results.

Conclusions: Parents who enrolled their newborn in a RCT of GS demonstrated awareness of a psychological risk, but generally held positive beliefs about GS and perceived the benefits outweighed the risk.

Genetics in Medicine (2020) 22:416-422; https://doi.org/10.1038/s41436019-0644-5

Keywords: genome sequencing; newborn; attitudes; risks; utility

\section{INTRODUCTION}

Genome sequencing (GS) is now clinically available for the diagnosis of suspected genetic conditions in children and can lead to a molecular diagnosis that, in some cases, may alter medical treatment and decision making. ${ }^{1-3}$ Many clinicians, bioethicists, and payers have concerns about both the clinical utility of GS and the ethical and social issues that may arise for patients and families, particularly when testing is used in the neonatal population. Clinicians caring for critically ill children have differing opinions on the usefulness of GS results for clinical care and concerns about the inability to interpret many findings from GS that may cause uncertainty or confusion for families. ${ }^{4,5}$ Clinicians and ethicists have also raised concerns about potential harms to patients and families through unwelcome information, parental blame, breaches of genetic privacy, discrimination, moral distress over the use of or waiting on GS to facilitate life-limiting decisions, and societal stigmatization of individuals with disabilities. ${ }^{4-7}$

While some parents of infants have shared concerns about privacy and unfavorable results, ${ }^{8}$ this is reported to occur at lower rates than in clinicians. ${ }^{9}$ This adds to concerns about whether parents are making adequately informed decisions. As GS moves into clinical use, particularly for vulnerable neonatal populations, it is unclear whether it should be used only in highly selective cases or more broadly. This must be informed by an understanding of the attitudes and experiences of patients and families. Here we report a retrospective mixed-methods study of parents of infants who enrolled in a GS study that explores attitudes about GS and impacts of diagnoses received through GS or clinical evaluation.

\section{MATERIALS AND METHODS}

\section{Participants}

Participants for this study were recruited from parents who were enrolled in the National Institutes of Health (NIH) sponsored study, "Prospective Randomized Trial of the Clinical Utility of Rapid Next Generation Sequencing in Acutely Ill Neonates." ${ }^{10}$ The randomized controlled trial (RCT) enrolled patients 4 months of age or younger with a suspected genetic condition, but no unifying molecular

\footnotetext{
${ }^{1}$ Center for Pediatric Genomic Medicine, Children's Mercy Kansas City, Kansas City, MO, USA; ${ }^{2}$ School of Medicine, University of Missouri-Kansas City, Kansas City, MO, USA ${ }^{3}$ Division of Developmental and Behavioral Sciences, Children's Mercy Kansas City, Kansas City, MO, USA; ${ }^{4}$ Division of Health Services and Outcomes Research, Children's Mercy Kansas City, Kansas City, MO, USA; ${ }^{5}$ Bioethics Center, Children's Mercy Kansas City, Kansas City, MO, USA. Correspondence: Courtney Berrios (cdberrios@cmh.edu)
} 
or clinical diagnosis. RCT participants in the control arm $(n=33)$ and 30 of the GS arm $(n=32)$ received standard diagnostic testing as clinically indicated, and the GS arm also received trio-based rapid GS. ${ }^{10}$ All samples were retained in a genetic research repository for possible future study. Parents were also enrolled in the RCT, and were eligible for the current retrospective study if they spoke English, had no contact restrictions in the medical record, and had not participated or declined participation in an earlier prospective survey of psychosocial and ethical aspects of GS in this population. Contact by phone was attempted for each eligible parent using phone numbers in the institutional medical record. If contact was made, a verbal consent script was reviewed. This study was approved by the Children's Mercy Institutional Review Board.

\section{Surveys}

Parents could choose to complete the survey verbally by phone or receive an email link to complete it online. Responses were entered into a REDCap electronic database hosted at Children's Mercy. ${ }^{11}$ Sociodemographics collected included age, gender, marital status, number of children, race, ethnicity, highest level of education, and religiosity. Questions developed for this study included 5-point Likert scale questions (strongly disagree to strongly agree) addressing three attitudes: (1) genetic testing as an opportunity, (2) that results might be disturbing, and (3) willingness to enroll child in future genetic research (full questions in Fig. 1). Five-point Likert-type scale questions (not at all, slightly, somewhat, a lot, extremely) were also used to assess how much participants felt blameworthy, confused, hopeful, disappointed, at fault, and uncertain during their infant's hospitalization when GS RCT participation was offered.

The survey also included validated scales discussed briefly here with details provided in the supplementary material

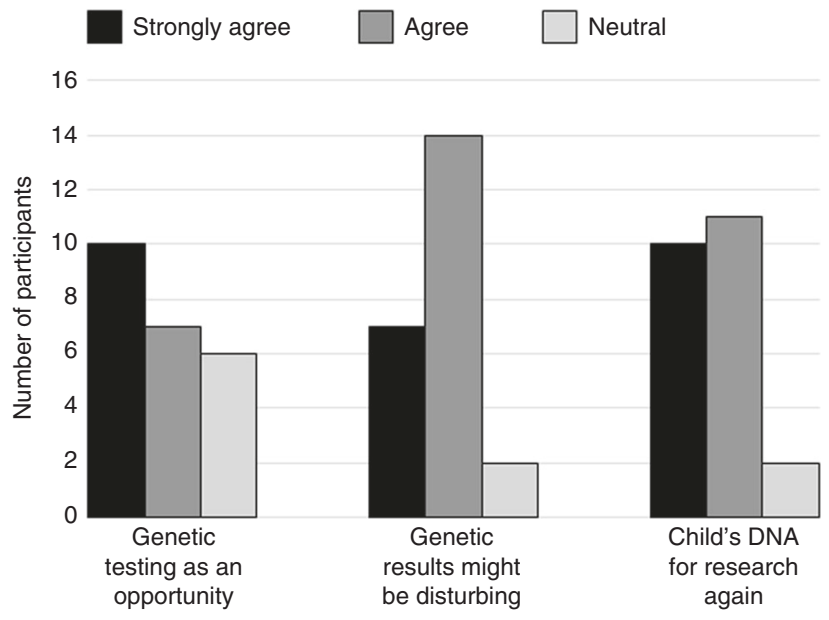

Fig. 1 Participant responses to survey Likert scale questions about attitudes. Full survey statements were as follows: (1) Genetic testing is an opportunity to get information that will help me improve my child's health, (2) Genetic testing might get some results that would be disturbing, and (3) I would allow my child's DNA to be used for research again.
(Supplementary Methods). Genetic literacy and applied genetic knowledge were measured by the awareness subscale of the Genetic Literacy and Comprehension (GLAC) instrument ${ }^{12}$ and the applied subscale of Fitzgerald-Butt et al.'s genetic knowledge measure, ${ }^{13}$ respectively. Numeracy was measured using the Subjective Numeracy Scale, which measures an individual's beliefs about their math skills and preferences regarding the presentation of information. ${ }^{14}$ Lastly, the Hospital Anxiety and Depression Scale (HADS) measured the presence and severity of anxiety and depression. ${ }^{15}$ For each scale, higher scores indicate higher levels of genetic literacy, genetic knowledge, numeracy, or symptoms of anxiety or depression.

\section{Interviews}

Study interviews were completed by phone and followed a semistructured interview guide (Supplementary Methods). Questions included memories about the purpose of the study, the process of enrollment, parents' attitudes about being approached about GS and research for their infant, and worries about enrolling in GS research. We also asked whether other genetic testing had been completed, whether a genetic diagnosis was received, the impact of the diagnosis (if applicable), and their general attitudes about genomic testing. Parents were asked to categorize their attitudes about having GS and research suggested for their child as negative, positive, or neutral and their general feelings about genomic testing as beneficial, harmful, or neither. These were followed by probes for explanation. All other interview questions were open-ended.

\section{Clinical and RCT data}

The institution's medical record for each participant's child enrolled in the RCT was reviewed to record whether a unifying diagnosis was identified and what clinical genetic tests had been completed. If a diagnosis was identified, the specific diagnosis and its modality (clinical or molecularly confirmed and whether it was obtained through the RCT or clinical testing) were recorded. The child's arm in the RCT (GS or control) was recorded.

\section{Analysis}

Survey data was imported into SPSS version $24^{16}$ for statistical analysis. Descriptive statistics were calculated for all variables. Fisher's exact tests were used to test for differences in attitudes by sociodemographic groups, feelings while in the neonatal intensive care unit (NICU), and whether a diagnosis was received. Mann-Whitney $U$ tests were used to test for differences in attitudes by genetic literacy, genetic knowledge, numeracy, anxiety and depression, and to test for differences in anxiety and depression by whether or not a diagnosis was received. Reported $p$ values are two-sided. All study interviews were recorded and transcribed. Initial codes were developed based on interview guide topics and an initial reading of study interviews. Five transcripts were coded by two study team members who discussed discrepancies, revised the codebook 
to include emerging themes, and developed rules for consistent coding. Each transcript was then coded by two independent coders. Codes were compared and discrepancies addressed until consensus was reached. Coded text was organized and analyzed for themes.

\section{Population}

\section{RESULTS}

One hundred twenty-eight parents (from 65 families) were enrolled in the RCT of sequencing in newborns. Of these, 40 individuals were ineligible for enrollment in the retrospective study reported herein because they had previously participated in or declined participation in a survey about psychosocial aspects of GS or were determined to be ineligible due to language or contact restrictions. From the 88 eligible parents (from 45 families) contact was made with 30;39 did not respond to voicemails, 5 did not respond to messages left with the other parent, and 14 could not be reached with available contact information. From the 30 parents contacted, 3 declined participation, and 4 verbally consented to participation but did not complete any study activities. A total of 23 individuals (parents of 19 different newborns) completed the survey and 22 also completed the interview between May 2017 and February 2018 (1-3 years after RCT enrollment). Demographics of the participants are presented in Table $\mathbf{1 .}$

The mean score for genetic literacy (GLAC) $(\mu=5.39$, $\mathrm{SD}=1.38)$ was comparable with that of a population-based consumer panel $(\mu=4.98, \mathrm{SD}=1.76)^{17}$ and an adult gastroenterology clinic population $(\mu=5.9, \mathrm{SD}=1.2){ }^{12}$ Applied knowledge of genetics was high with a median score of 10.0 (interquartile range $[\mathrm{IQR}]=9.0-10.0)$ on a 10 -point scale. Subjective numeracy (median $=4.13, \mathrm{IQR}=3.75-4.50)$ shows a similar median to that identified in a population-based validation study of $>800$ individuals (median $=4.2$, IQR $3.2-4.8) .{ }^{18}$

\section{Attitudes}

When asked to categorize their feelings at the time they heard that doctors wanted to consider GS for their child, 13 recalled feeling positive, 4 neutral, and 2 negative ( 3 interviewed did not categorize their feelings). No statistically significant associations were found between these categories of feelings about being approached about GS and reported feelings in the NICU (blameworthy, confused, hopeful, disappointed, at fault, uncertain). Positive feelings were most often around hope of a diagnostic answer that would allow them and doctors to understand the reasons for their child's symptoms. Parents said that they wanted to be thorough and do anything to help their child. When speaking of potential answers for their child, parents sometimes flowed into discussions of altruistic motivations related to the research study.

"I think we were encouraged because it felt like we were being very thorough, or the physicians were being very thorough.... And of course we were optimistic that it might
Table 1 Participant characteristics

Total $(n=23)$

$n(\%)$

\begin{tabular}{lc}
\hline NSIGHT study arm & $12(52.2)$ \\
\hline Sequencing & $10(43.5)$ \\
\hline Control & $1(4.3)$ \\
\hline Crossover from control to sequencing & \\
\hline Participant age (years) & $4(17.4)$ \\
\hline $20-29$ & $14(60.9)$ \\
\hline $30-39$ & $3(13.0)$ \\
\hline $40+$ & $2(8.7)$ \\
\hline No response & $17(73.9)$ \\
\hline Participant gender & $6(26.1)$ \\
\hline Female & \\
\hline Male & $20(87.0)$ \\
\hline Marital status & $3(13.0)$ \\
\hline Married & $9(39.1)$ \\
\hline Single & \\
\hline Education & $14(60.9)$ \\
\hline $\begin{array}{l}\text { Some high school/graduated high school/some } \\
\text { college }\end{array}$ & $11(47.8)$ \\
\hline $\begin{array}{l}\text { Graduated college/master's degree/ } \\
\text { doctoral degree }\end{array}$ & $0(0)$ \\
\hline Race & $21(91.3)$ \\
\hline White & $19(65.2)$ \\
\hline Black/African American or other & $4(17.4)$ \\
\hline Ethnicity & \\
\hline Hispanic/Latino & \\
\hline Not Hispanic/not Latino & \\
\hline Sory rer of children & \\
\hline Not at all religious & \\
\hline
\end{tabular}

give us some kind of answer that we weren't getting from other testing."

"I was just hopeful that maybe they'd be able to find something that gave them an answer. Not maybe just for her but for any child in the future, you know as they were just sending the genetics."

Parents who reported negative or neutral feelings also spoke of hope for an answer for their child, but additionally relayed feelings that the nomination for GS brought the realization that something was "really wrong" with their child. More negative feelings were also related to fears about what the results would reveal about their child's illness and future. 
"It was a big deal. It made it more real that we did not have a perfectly normal, healthy baby. So I guess it was a reality check."

"It needed to be done, but I was scared of the unknown."

A majority interviewed (59\%) responded that they had no worries about enrolling in a study that included GS. However, other participants revealed worries such as potential costs of the testing, another needle stick for their child, being randomized to the control group, or misuse of their child's information. One parent noted that their worries may have been lower due to the easy availability of genetic testing, particularly direct-to-consumer testing, that normalizes the experience. The most common worry was of receiving bad news related to their baby's prognosis or treatment.

"The only worry I think that I had was that they were going to find something that was going to be life-threatening for him or they were going to find that they couldn't help him."

In comparison, parents' attitudes at the time of this study's survey and interview were more uniformly positive than their recollection of their thoughts when the GS RCT was offered. Most parents (74\%) agreed that genetic testing was an opportunity to gain information that would improve their child's health (Fig. 1). There were no statistically significant differences in this attitude between sociodemographic groups, arm of the RCT, or by scores for genetic literacy, genetic knowledge, numeracy, anxiety, or depression.

When asked if they felt GS was generally beneficial, neutral, or harmful, all parents indicated that they felt it was beneficial. Participants reported that it allowed parents to have more information and that "being educated is always good." They also noted it could provide guidance on treatment or prognosis and clarify recurrence risks. Participants also noted altruistic benefits in helping other children and families. Some participants noted that GS was beneficial only if a diagnosis was received. Some noted that GS may produce information that parents did not want, particularly about a poor prognosis. There was also mention of concerns over equitable use of health-care dollars. No differences in themes were noted between RCT study arms. Multiple participants also qualified their response in that they felt that GS was beneficial for children with unexplained medical conditions, but that they would not recommend it for healthy children.

"I don't think that it's something that I would choose for my healthy kids unless there was a reason to do it. But in cases where you are trying to get an answer that you couldn't really get any other way I think there's absolutely good reason for it. I know that there are ethical concerns that are attached to it, and when you research the discussions people have strong feelings on those as well, but I think in our particular case and in cases similar to ours I think the benefits of doing this type of studies significantly outweigh the risks or any other concerns that might be there."

This recognition of risks was also reflected in the survey data as 21 of $23(91 \%)$ agreed or strongly agreed that genetic testing could produce results that would be disturbing (Fig. 1). Rates of this widely held belief did not differ by sociodemographic group, nor was it associated with scores on the genetic literacy, genetic knowledge, numeracy, anxiety, or depression scales. Despite this recognition of risk, 21 of 23 (91\%) also agreed or strongly agreed that they would let their child's DNA be used for research again. Participants identifying as White were more likely than those identifying as any other race to agree or strongly agree that they would let their child's DNA be used for research again (100\% vs. $50 \%$, respectively, Fisher's exact test, $p=0.024)$. There were no other differences by sociodemographic groups or scores for genetic literacy, genetic knowledge, numeracy, anxiety, or depression.

\section{Impact of a diagnosis}

Of the 23 participants, medical record review revealed that 9 had received a molecularly confirmed diagnosis for their child (4 through the RCT and 5 through standard clinical genetic testing) (Fig. 2). An additional three had received a unifying diagnosis for their child's symptoms through a clinical syndromic diagnosis. Secondary findings were not reported. All individuals who had received a molecular or clinical diagnosis per the electronic medical record (EMR) recalled receiving the diagnosis. An additional two participants also recalled receiving a diagnosis when none was identified in our institution's medical record. There was some discrepancy between the medical record and the participant's recollection about whether a molecular result came through the RCT or clinical genetic testing, and some indicated they were not certain which source the result came from.

Regardless of the source or type of result, most participants expressed benefits of receiving a diagnosis. Participants referred to the diagnosis as an answer for their child's illness that provided clarity. This answer was perceived to have downstream effects that included both changes to their child's medical care and personal utility. Themes of benefits discussed by participants are listed in Table 2 with exemplary participant quotes. Individual participants often expressed multiple ways in which a diagnosis was impactful, and these responses support the value of receiving a diagnosis beyond traditional views of clinical utility. One parent expressed that their medical team questioned the family's desire for a diagnosis when it was not expected to inform treatment decisions.

"We were asked at that time by the team why it was so important to have a diagnosis to explain. It wouldn't change the care. And I think as parents, what that did is it gave us reassurance that as we made decisions for him we really knew what was going on. And it also put a name to it, you 


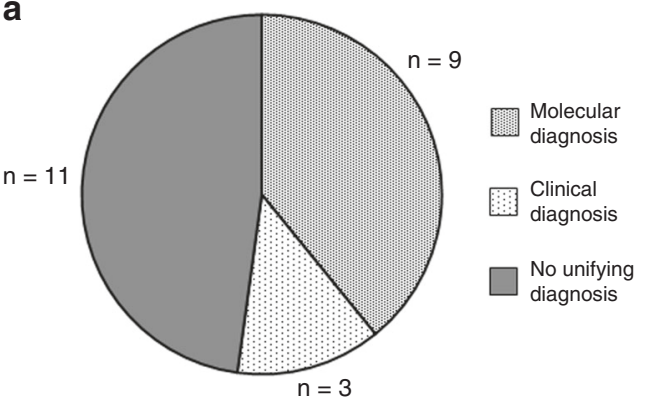

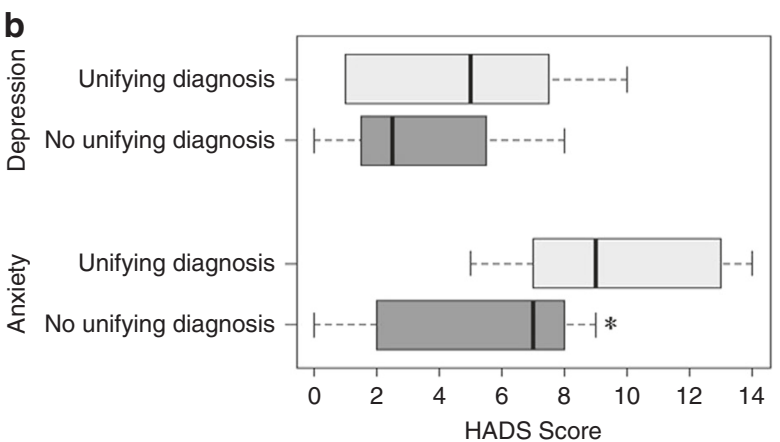

Fig. 2 Diagnostic status of child and association with parental Hospital Anxiety and Depression (HADS) subscale scores. (a) Diagnostic status of the children of study participants. (b) Distribution of HADS subscale scores for participants whose child has received a unifying diagnosis (molecular or clinical diagnosis) compared to those who have not received a unifying diagnosis for their child's symptoms. Boxes represent the interquartile range and whiskers the minimum and maximum scores. *Statistically significantly different by Mann-Whitney $U$ test.

Table 2 Participants' expressed benefits of receiving a diagnosis

Benefit of diagnosis Exemplary quote

\section{Changes in medical care}

Additional screening "So they knew that they needed to keep

recommendations

digging and look to see if he did or didn't

have it. And after more imaging they found he indeed did have one more condition."

\section{Guiding treatment \\ "Her diagnosis is [specific variant] and so} decisions using that as the starting point for medicines and things like that gave us a way to start moving forward, like what medicines to start her on."

Redirection to "Having the diagnosis and knowing that it palliative care was something that was not treatable almost, you know, gave my wife and I that closure to know that it was OK for us to explore that aspect of the care as well.... We're not giving up on him. We know what's wrong with him and we're making the best decision we can based on that information."

\section{Personal utility}

Chance to find information and "It just helped me do some research online support Preparation for the future

Recurrence risk information

Removal of guilt know, versus saying our son has these symptoms and we don't really know why. It gave us some closure as parents to know that there was a reason, that it's been identified, and we can put a name to it, and network with other people who were impacted by similar things. I think there were a lot of ways that it benefited us as parents."

In some cases, receiving a diagnosis provided parents with a more positive outlook for their child's future as they came to understand how symptoms may evolve with time or what treatment options may be best.

"Without the results, just from what he looked like, he was just very floppy, lethargic, not interacting with his environment at all. These people with this disorder, we know, they develop and they can live independent lives. It just changed my outlook. I became more positive about it."

Others discussed receiving information that was upsetting and gave them a more negative outlook.

"Well, I guess it made me feel a little worse, because frankly, the gene with the mutation comes in kiddos with more severe delays."

Still others became acutely aware of the current limitations of genetic knowledge as they realized the limited information that is available about some genetic conditions.

"It gave a name to her specific epilepsy, which was good, but at the same time, what we were told and what I found through further research is there are not very many people who have her specific diagnosis. So it continued to open up new questions that we have not necessarily gotten answers to."

"Well, his situation was a little bit different because his symptoms do not fit nicely in this disorder's description. So it helps to understand part of what was going on, but then 
there is a whole other aspect of what's going on with him that is very confusing."

There were no statistically significant differences in attitudes about genetic testing depending on whether they had received a diagnosis for their child. This remained true if separated by molecular or clinical diagnosis, or by the parent's perception of whether or not their child had received a diagnosis. HADS depression subscale scores also did not show statistically significant differences between participants who had received a diagnosis for their child (median $=5.0$, $\mathrm{IQR}=1.0-7.5)$ and those who had not (median $=2.5, \mathrm{IQR}=$ 1.5-5.5) (Mann-Whitney $U=34.50, p=0.427$ ) (Fig. 2). However, HADS anxiety subscales scores were statistically significantly higher in those who had received a diagnosis (median $=9.0, \mathrm{IQR}=7.0-13.0)$ than in those who had not (median $=7.0, \quad \mathrm{IQR}=2.0-8.0) \quad($ Mann-Whitney $U=20.50$, $p=0.026)$ (Fig. 2).

\section{DISCUSSION}

In this small and selected population, most participants reported positive responses to being offered GS for their infant largely due to hopes of receiving an explanation for their child's medical concerns. Their positive attitudes about the ability of GS to provide beneficial information continued to the time of this retrospective study, long after their infants had been discharged from the NICU. While just under half of participants volunteered worries when asked in an openended manner, $>90 \%$ recognized the possibility of psychosocial risks of the information when asked directly. This suggests participants simultaneously carried both hope that GS can provide answers, and the recognition that it involved risks. Furthermore, most participants who had received a unifying diagnosis for the child reported benefits of clinical and/or personal utility, while this was tempered for some by results that provided negative prognoses for their child or a forced recognition of the current limitations of genetic medicine.

The results of this study fit with the largely positive views of genetic testing among the US population that have been identified in previous research. ${ }^{19}$ Particularly, our findings highlight that parents who have consented to allow their hospitalized newborns to undergo GS have mostly positive views of GS as diagnostic test. They hope that it will provide an answer for their child's medical condition that will be impactful for their child's care and for family planning, as has been reported in parents of a pediatric cohort of varied ages. ${ }^{20}$

Concern has been raised that parents do not adequately understand other risks of GS research. ${ }^{21}$ The comment by a participant in this study that risks may have been normalized by the widespread availability of consumer-driven genetic testing may suggest that some risks are not seen as seriously by patients and families as they are by clinicians. However, while previous research had identified the worry of unfavorable results and awareness of risk of disturbing news in those declining GS for their infant, ${ }^{8}$ this study demonstrates the presence of these concerns also in parents who consent to GS for their child. Indeed, the spontaneous mention of risks by nearly half of our participants and the qualification by several that their positive view of GS was dependent upon the context for their child's health suggest these participants had a measured approach to weighing benefits and risks based on their child's particular situation. While we cannot ignore that this is a vulnerable population, they do not appear to have been naive about risks of GS, but to have judged that the potential benefits outweighed those risks for this child.

For those participants who received a diagnosis for their infant, either clinical or molecular, a value of the diagnosis beyond typical views of clinical utility was shared. This substantiates other studies recognizing that factors such as future planning, reduced worry or guilt, and access to support networks and disability services are valued by parents. ${ }^{22-26}$ By the enrollment criteria of the RCT, participants in this study were early in the journey of their child's illness at the time a diagnosis was sought, which may reflect the higher perceived importance of a diagnosis that has been reported for families earlier in the diagnostic odyssey. ${ }^{27}$ Previous studies have also reported limitations of receiving a diagnosis including a loss of hope for recovery, frustration about limited information on a diagnosis, and a sense of isolation in a rare diagnosis that were also shared by our participants. ${ }^{24,28}$ The higher level of anxiety seen here in parents of children who had received a diagnosis is concerning, but given the small sample size, needs further study. Yet despite some with high anxiety levels and the limitations participants identified in GS, all participants, even those not receiving a diagnosis, still reported that they felt GS was beneficial. While patient and family expectations for both the likelihood of a diagnosis and its impact on their child's care are essential to manage in the setting of GS, ${ }^{29}$ clinicians also must consider the expanded views of benefits expressed by parents when considering potential utility of genomic testing.

The conclusions of this study are limited by the highly selected nature of the sample. Participants in the RCT were selected by clinicians for nomination to the study and by consent to the trial. Furthermore, participants in the study reported here are a further subset of that group, selected by their response to attempted contact for this retrospective study and consent to participation. Comparisons by RCT arm may have been limited by retaining samples from both arms in a genetic research repository. Racial and ethnic diversity in the sample was also limited. Participants demonstrated genetic literacy and subjective numeracy scores comparable with other populations, but had high levels of applied genetics knowledge, which may be a function of their experiences in the RCT and clinical genetic evaluations. Furthermore, given the limited population from which recruitment was available, statistical power calculations were not completed before the study to determine a needed sample size for quantitative analyses. The small sample may not be powered to detect some differences in attitudes about GS based on participant characteristics. Lastly, participants completed this study's survey and interview between 1 and 3 years after enrollment 
in the RCT. The retrospective nature of data collection and time lapse may have created a recall bias for questions asking about their feelings at enrollment in the GS RCT.

As GS becomes more widely adopted for clinical care, the question of when it should be used has become increasingly urgent for clinicians and payers. Recent guidance statements use impact on medical care and ethical concerns to propose that at the current time the use of GS for diagnostic purposes in newborns is warranted while screening use is not. ${ }^{30,31}$ The parental attitudes reported here support the use of GS as a diagnostic tool in the newborn population. Furthermore, while parents felt that the possible benefits outweighed the risks for their infants, some noted that this may not always be the case, especially for healthy children. Both studies of clinical utility and parental attitudes and experiences with genomic testing in newborns in a screening context will be essential in understanding how genomic technologies should be used in the future.

\section{SUPPLEMENTARY INFORMATION}

The online version of this article (https://doi.org/10.1038/s41436019-0644-5) contains supplementary material, which is available to authorized users.

\section{ACKNOWLEDGEMENTS}

We thank the study participants for their time and willingness to share their views and experiences. This study was funded by a grant from the US National Human Genome Research Institute U19 grant U19HD077693 (sub-award agreement 3282-S1-A2).

\section{DISCLOSURE}

The authors declare no conflicts of interest.

Publisher's note: Springer Nature remains neutral with regard to jurisdictional claims in published maps and institutional affiliations.

\section{REFERENCES}

1. Meng L, Pammi M, Saronwala A, et al. Use of exome sequencing for infants in intensive care units: ascertainment of severe single-gene disorders and effect on medical management. JAMA Pediatr. 2017;171: e173438.

2. Stark Z, Tan TY, Chong B, et al. A prospective evaluation of whole-exome sequencing as a first-tier molecular test in infants with suspected monogenic disorders. Genet Med. 2016;18:1090-1096.

3. Willig LK, Petrikin JE, Smith LD, et al. Whole-genome sequencing for identification of Mendelian disorders in critically ill infants: a retrospective analysis of diagnostic and clinical findings. Lancet Respir Med. 2015; 3:377-387.

4. Knapp B, Decker C, Lantos JD. Neonatologists' attitudes about diagnostic whole-genome sequencing in the NICU. Pediatrics. 2019;143 suppl 1: S54-S57.

5. Char DS, Lee SS, Magnus D, Cho M. Anticipating uncertainty and irrevocable decisions: provider perspectives on implementing wholegenome sequencing in critically ill children with heart disease. Genet Med. 2018;20:1455-1461.

6. Deem MJ. Whole-genome sequencing and disability in the NICU: exploring practical and ethical challenges. Pediatrics. 2016;137 suppl 1: S47-55.

7. Wilkinson DJ, Barnett C, Savulescu J, Newson AJ. Genomic intensive care: should we perform genome testing in critically ill newborns? Arch Dis Child Fetal Neonatal Ed. 2016;101:F94-98.
8. Genetti CA, Schwartz TS, Robinson JO, et al. Parental interest in genomic sequencing of newborns: enrollment experience from the BabySeq Project. Genet Med. 2019;21:622-630.

9. Pereira S, Robinson JO, Gutierrez AM, et al. Perceived benefits, risks, and utility of newborn genomic sequencing in the BabySeq Project. Pediatrics. 2019;143 suppl 1:S6-S13.

10. Petrikin JE, Cakici JA, Clark MM, et al. The NSIGHT1-randomized controlled trial: rapid whole-genome sequencing for accelerated etiologic diagnosis in critically ill infants. NPJ Genom Med. 2018;3:6.

11. Harris PATR, Thielke R, Payne J, Gonzalez N, Conde JG. Research electronic data capture (REDCap) - a metadata-driven methodology and workflow process for providing translational research informatics support. J Biomed Inform. 2009;42:377-381.

12. Hooker GW, Peay H, Erby L, Bayless T, Biesecker BB, Roter DL. Genetic literacy and patient perceptions of IBD testing utility and disease control: a randomized vignette study of genetic testing. Inflamm Bowel Dis. 2014;20:901-908.

13. Fitzgerald-Butt SM, Bodine A, Fry KM, et al. Measuring genetic knowledge: a brief survey instrument for adolescents and adults. Clin Genet. 2016;89:235-243.

14. Fagerlin A, Zikmund-Fisher BJ, Ubel PA, Jankovic A, Derry HA, Smith DM. Measuring numeracy without a math test: development of the Subjective Numeracy Scale. Medical Decis Making. 2007;27:672-680.

15. Zigmond AS, Snaith RP. The hospital anxiety and depression scale. Acta Psychiatr Scand. 1983;67:361-370.

16. IBM Corp. SPSS Statistics for Windows, Version 24.0. Armonk, NY: IBM Corp. 2016.

17. Abrams LR, McBride CM, Hooker GW, Cappella JN, Koehly LM. The many facets of genetic literacy: assessing the scalability of multiple measures for broad use in survey research. PLoS ONE. 2015;10:e0141532.

18. Zikmund-Fisher BJ, Smith DM, Ubel PA, Fagerlin A. Validation of the subjective numeracy scale: effects of low numeracy on comprehension of risk communications and utility elicitations. Med Decis Making. 2007; 27:663-671.

19. LePoire E, Basu B, Walker L, Bowen DJ. What do people think about genetics? A systematic review. J Community Genet. 2019;10:171-187.

20. Anderson JA, Meyn MS, Shuman C, et al. Parents perspectives on whole genome sequencing for their children: qualified enthusiasm? J Med Ethics. 2017:43:535-539.

21. Klima J, Fitzgerald-Butt SM, Kelleher KJ, et al. Understanding of informed consent by parents of children enrolled in a genetic biobank. Genet Med. 2014;16:141-148.

22. Carmichael N, Tsipis J, Windmueller G, Mandel L, Estrella E. "Is it going to hurt?": the impact of the diagnostic odyssey on children and their families. J Genet Couns. 2015;24:325-335.

23. Reiff M, Giarelli E, Bernhardt BA, et al. Parents' perceptions of the usefulness of chromosomal microarray analysis for children with autism spectrum disorders. J Autism Dev Disord. 2015;45:3262-3275.

24. Rosell AM, Pena LD, Schoch K, et al. Not the end of the odyssey: parental perceptions of whole exome sequencing (WES) in pediatric undiagnosed disorders. J Genet Couns. 2016;25:1019-1031.

25. Stivers T, Timmermans $S$. The actionability of exome sequencing testing results. Sociol Health Illn. 2017;39:1542-1556.

26. Malek J, Slashinski MJ, Robinson JO, et al. Parental perspectives on whole-exome sequencing in pediatric cancer: a typology of perceived utility. JCO Precis Oncol. 2017;1:1-10.

27. Makela NL, Birch PH, Friedman JM, Marra CA. Parental perceived value of a diagnosis for intellectual disability (ID): a qualitative comparison of families with and without a diagnosis for their child's ID. Am J Med Genet A. 2009;149A:2393-2402.

28. Krabbenborg L, Vissers LE, Schieving J, et al. Understanding the psychosocial effects of WES test results on parents of children with rare diseases. J Genet Couns. 2016;25:1207-1214.

29. Wynn J, Lewis K, Amendola LM, et al. Clinical providers' experiences with returning results from genomic sequencing: an interview study. BMC Med Genomics. 2018;11:45.

30. Johnston J, Lantos JD, Goldenberg A, et al. Sequencing newborns: a call for nuanced use of genomic technologies. Hastings Cent Rep. 2018; 48 suppl 2:S2-S6.

31. Borghesi A, Mencarelli MA, Memo L, et al. Intersociety policy statement on the use of whole-exome sequencing in the critically ill newborn infant. Ital J Pediatr. 2017:43:100. 